\title{
On the initial-value problems with data on a double characteristic
}

By

\author{
Yukiko Hasegawa
}

(Received December 10, 1970)

\section{$\S 1$. Introduction}

Let us consider a linear partial differential equation

$$
\left.a(x, \partial) u(x) \equiv \sum_{|\nu| \leqslant m} a_{\nu}(x) \partial^{\nu} u(x)=f(x), x \in R^{n} .{ }^{*}\right)
$$

Let $S$ be a hypersurface in $R^{n}$ defined in a neighborhood of $x_{0} \in S$ by

$$
\varphi(x)=0 ; \varphi_{x}(x) \equiv\left(\partial_{1} \varphi(x), \partial_{2} \varphi(x), \ldots, \partial_{n} \varphi(x)\right) \neq 0
$$

We say that $S$ is a double characteristic hypersurface of the operator $a(x, \partial)$, if $\varphi$ satisfies the following conditions:

$$
\left\{\begin{array}{l}
h\left(x, \varphi_{x}\right)=0, \quad x \in S, \\
\frac{\partial}{\partial \xi_{i}} h\left(x, \varphi_{x}\right)=0, \quad x \in S, i=1,2, \ldots, n, \\
\sum_{i, j}\left|\frac{\partial^{2}}{\partial \xi_{i} d \xi_{j}} h\left(x, \varphi_{x}\right)\right| \neq 0, \quad x \in S,
\end{array}\right.
$$

where $h(x, \xi)=\sum_{|\nu|=m} a_{\nu}(x) \xi^{\nu}$.

*) In this article we use the following notations:

$\partial$ stands for $\frac{\partial}{\partial x}$ and $\partial_{i}, \partial_{r}, \partial_{y}$ stand for $\frac{\partial}{\partial x_{i}}, \frac{\partial}{\partial x}$ and $\frac{\partial}{\partial y}$ respectively. In the case where $\partial_{y}^{\prime} u(y)$, we often represent it simply by $\partial^{\alpha} u(y)$. 
In this case the initial-value problem of (1.1) with data on $S$ can be reduced to the following initial-value problem of (1.4) with data on $x=0\left(x \in R^{1}\right)$, by a suitable change of variables

$$
\begin{aligned}
& x a_{m, 0}(x, y) \partial_{x}^{m} u+\sum_{\mid \alpha\}=1} x a_{m-1, \alpha}(x, y) \partial_{x}^{m-1} \partial_{y}^{\alpha} u+a_{m-1,0}(x, y) \partial_{x}^{m-1} u \\
& \quad+\sum_{i \leqslant m-2,|\alpha|+i \leqslant m} a_{i, \alpha}(x, y) \partial_{x}^{i} \partial_{y}^{\alpha} u=f(x, y),
\end{aligned}
$$

where $x \in R^{1}, y \in R^{n-1}, \sum_{|\alpha|=2}\left|a_{m-2, \alpha}(0,0)\right| \neq 0$.

Now, our problem is the initial-value problem of (1.4) with data on the hypersurface $x=0$. In this article we treat our problem only within the class of real analytic functions, more precisely we assume that the coefficients, initial data and solutions are all real analytic in the neighborhood of the origin.

At first let us observe the following fact:

If there are given the initial data

$$
\partial_{x}^{j} u(0, y)=u_{j}(y), \quad j=0,1, \ldots, m-3,
$$

there exists a solution $u$ of (1.4), and such a solution is not unique.

In fact, there exists a real non-zero vector $\eta=\left(\eta_{1}, \ldots, \eta_{n-1}\right)$ such that

$$
\sum_{|\alpha|=2}\left[a_{m-2, \alpha}(0,0)_{1}^{1} \eta^{\alpha} \neq 0\right.
$$

and we consider the following Goursat problem to (1.4):

$$
\left\{\begin{array}{l}
\partial_{x}^{j}\left(u-\varphi_{m-3}\right)(0, y)=0 \quad(j=0,1,2, \ldots, m-3) \\
<\partial, \eta>^{j}\left(u-\varphi_{m-3}\right)_{<y, \eta>=0}=0 \quad(j=0,1)
\end{array}\right.
$$

where $\varphi_{m-3}=\sum_{p=0}^{m-3} u_{p}(y) x^{p} / p ! ;\langle\partial, \eta\rangle=\eta_{1} \frac{\partial}{\partial y_{1}}+\cdots+\eta_{n-1} \frac{\partial}{\partial y_{n-1}}$. This $u(x, y)$ gives surely a desired solution of the problem (1.4)-(1.5).

Concerning the Goursat problem, we refer to Hörmander [2], 
p. $116-119 * *)$

Let us consider further the following initial data

$$
\partial_{x}^{j} u(0, y)=u_{j}(y), \quad j=0,1,2, \ldots, m-2
$$

or

$$
\partial_{x}^{j} u(0, y)=u_{j}(y), \quad j=0,1,2, \ldots, m-1 .
$$

In these cases, the solution does not exist always without some additional conditions.

At first, let us consider the problem (1.4)-(1.6) in the case where

$$
a_{m, 0}(0, y) \equiv a_{m-1,0}(0, y) \equiv 0 .
$$

The necessary condition for the existence is easily obtained as follows: Let

$$
u(x, y)=\sum_{p \geqslant 0} u_{p}(y) x^{p} / p !
$$

be a solution, then putting this in (1.4), and comparing the coefficients of $x^{0}$, we have

$$
\sum_{\substack{i \leqslant m-2 \\ i+|\alpha| \leqslant m}} a_{i, \alpha}(0, y) \partial_{x}^{i} \partial_{y}^{\alpha} \varphi(0, y)=f(0, y)
$$

where

$$
\varphi(x, y)=\sum_{p=0}^{m-2} u_{p}(y) x^{p} / p !
$$

Let us show that this condition is also sufficient. For this purpose,

**) We note that Theorem 5.1.1' still holds when defining $A$ as the set of multi-indices in the sum on the right-hand side of $(5.1 .1)$ such that $a^{\alpha}(0) \neq 0$.

In fact, when $A$ is thus defined, instead of

$$
D^{\beta} U(z)=\sum_{\alpha \in \boldsymbol{A}} a^{\alpha}\left(z^{\prime}\right) e^{\lambda(\rho(\alpha)-\rho(\beta))} D^{\alpha} U(z)+F(z),
$$

we consider the following equation:

$$
\begin{aligned}
D^{\beta} U(z) & =\sum_{\alpha \in A} a^{\alpha}\left(z^{\prime}\right) e^{\lambda(\rho(\alpha)-\rho(\beta))} D^{\alpha} U(z) \\
& +\sum_{\substack{|\alpha|<|\beta| \\
\alpha \in \mathbb{E} .1}} a^{\alpha}\left(z^{\prime}\right) e^{\lambda(\rho(\alpha)-\rho(\beta))} D^{\alpha} U(z)+F(z),
\end{aligned}
$$

Where all the coefficients $a^{\prime \prime}\left(z^{\prime}\right)$ appearing in the second summation vanish at the origin. Thus we can claim the same existance theorem under this assumption. 
let $u_{0}(x, y)$ be the solution of the following Goursat problem to (1.4) with data

$$
\left\{\begin{array}{l}
\partial_{x}^{j}\left(u_{0}-\varphi\right)(0, y)=0 \quad(j=0,1,2, \ldots, m-3) \\
\langle\partial, \eta\rangle^{j}\left(u_{0}-\varphi\right)_{<y, \eta\rangle=0}=0 \quad(j=0,1)
\end{array}\right.
$$

Now we can show that $\partial_{x}^{m-2} u_{0}(0, y) \equiv u_{m-2}(y)$. In fact, let

$$
\begin{aligned}
u_{0} & =\sum_{p=0}^{m-3} u_{p}(y) x^{p} / p !+\tilde{u}_{m-2}(y) x^{m-2} /(m-2) !+\sum_{p \geqslant m-1} u_{p}(y) x^{p} / p ! \\
& =\tilde{\varphi}_{m-2}+\underset{p \geqslant m-1}{\sum_{p}} u_{p}(y) x^{p} / p !
\end{aligned}
$$

then the same relation as (1.9) holds for $\tilde{\varphi}_{m-2}$.

This implies, denoting

$$
\begin{aligned}
& L(y, \partial)=\sum_{|\alpha| \leqslant 2} a_{m-2, \alpha}(0, y) \partial_{y}^{\alpha} . \\
& L(y, \partial) \tilde{u}_{m-2}(y)=L(y, \partial) u_{m-2}(y) .
\end{aligned}
$$

On the other hand,

$$
\begin{aligned}
& <\partial, \eta>^{j}\left(u_{0}-\varphi\right)=<\partial, \eta>^{j}\left(\tilde{u}_{m-2}-u_{m-2}\right) x^{m-2} /(m-2) ! \\
& \quad+\sum_{p \geqslant m-1}<\partial, \eta>^{j} u_{p}(y) x^{p} / p !
\end{aligned}
$$

implies

$$
\langle\partial, \eta\rangle^{j}\left(\tilde{u}_{m-2}-u_{m-2}\right)_{<y, \eta>=0}=0, \quad j=0,1 .
$$

This shows, together with (1.11), $\tilde{u}_{m-2}(y) \equiv u_{m-2}(y)$, which completes the proof. Finally, let us note that the solution is not unique.

The purpose of this article is to investigate the situations when we remove the condition (1.8).

\section{§2. Statements of Theorems}

Let us recall the condition (1.8). The following theorem shows that, if we assume $a_{m, 0}(0, y) \equiv 0$, then the condition $a_{m-1,0}(0, y) \equiv 0$ is a reasonable assumption. 
On the initial-value problems with data on a double characteristic 361

\section{Theorem 1.}

In the case where $a_{m, 0}(0, y) \equiv 0$, if $a_{m-1,0}(0, y) \neq 0$, the ploblem (1.4)-(1.6) has not always a solution; more precisely, there exists an initial data (1.6), such that the initial value problem (1.4)-(1.6) has no analytic solution in any neighborhood of the origin.

However, if we assume $a_{m, 0}(0,0) \neq 0$, we have the following analogue of fuchsian theorems.

\section{Theorem 2.}

In the case where $a_{m, 0}(0,0) \neq 0$ and moreover for all nonnegative integers $p$, we have $p a_{m, 0}(0,0)+a_{m-1,0}(0,0) \neq 0$, then there exists always a unique solution for the initial value problem (1.4)(1.6).

\section{Theorem 3.}

In the case where $a_{m, 0}(0,0) \neq 0$ and moreover if for some nonnegative integer $p_{0}$ we have $p_{0} a_{m, 0}(0, y)+a_{m-1,0}(0, y) \equiv 0$, then $a$ necessary and sufficient condition concerning the initial data for the existence of the solution of the problem (1.4)-(1.6) is the following compatibility condition:

$$
\begin{aligned}
& \sum_{\substack{k+p_{0}+m-1 \\
k \geqslant 1}} a_{m, 0}^{(k)}(y) \frac{u_{s}(y)}{(s-m) !}+\sum_{|\alpha|=1} \sum_{k+s=p_{0}+m-2} a_{m-1, \alpha}^{(k)}(y) \frac{\partial_{y}^{\alpha} u_{s}(y)}{(s-m+1) !} \\
& \quad+\sum_{\substack{k+s=p_{0}+m-1 \\
k \geqslant 1}} a_{m-1,0}^{(k)}(y) \frac{u_{s}(y)}{(s-m+1) !} \\
& \quad+\sum_{\substack{i \leqslant m-2 \\
i+|\alpha| \leqslant m}} \sum_{s=p_{0}+i} a_{i, \alpha}^{(k)}(y) \frac{\partial_{y}^{\alpha} u_{s}(y)}{(s-i) !}=f^{\left(p_{0}\right)}(y),
\end{aligned}
$$

where $a_{j, \beta}(x, y)=\sum_{k \geqslant 0} a_{j, \beta}^{(k)}(y) x^{k}, f(x, y)=\sum_{k \geqslant 0} f^{(k)}(y) x^{k}$ and $u_{m-1}, u_{m}, \ldots$, $u_{m+p_{0}-2}$ are uniquely determined by the initial data $\left\{u_{0}, u_{1}, \ldots, u_{m-2}\right\}$.

In this case the solution is not unique. 


\section{Remark.}

We note that in the case of (1.8), the problem (1.4)-(1.7) can be also reduced to the Goursat problem. In fact, in this case we choose $\varphi=\sum_{p=0}^{m-1} u_{p}(y) x^{p} / p$ !, and in addition to (1.9), we have only to impose the following second compatibility condition:

$$
\begin{aligned}
\sum_{|\alpha|=1} & a_{m-1, \alpha}(0, y) \partial^{\alpha} u_{m-1}(y)+\partial_{x} a_{m-1,0}(0, y) u_{m-1}(y) \\
& +\partial_{x}\left(\sum_{\substack{i \leqslant m-2 \\
i+|\alpha| \leqslant m}} a_{i, \alpha}(x, y) \partial_{x}^{i} \partial_{y}^{\alpha} \varphi(x, y)\right)_{x=0}=\partial_{x} f(0, y) .
\end{aligned}
$$

But Theorem 2 could not be reduced to the Goursat problem. In fact, in this case we do not have a similar relation as (1.9) concerning $u_{m-2}(y)$, so the similar reasoning could not be applied to this case.

\section{§3. Proof of Theorem 1}

At first we consider the case where

$$
a_{m-1,0}(0,0)=0, \quad a_{m-1,0}(0, y) \not \equiv 0 .
$$

In this case we can find easily the initial data which we assert. In fact, let

$$
u(x, y)=\sum_{p \geqslant m-2} u_{p}(y) x^{p} / p !
$$

be the solution of (1.4). Then, in view of the coefficients of $x^{0}$, we should have

$$
a_{m-1,0}(0, y) u_{m-1}(y)+\sum_{|\alpha| \leqslant 2} a_{m-2, \alpha}(0, y) \partial^{\alpha} u_{m-2}(y)=f(0, y) .
$$

If we put $y=0$, then

$$
\sum_{|\alpha| \leqslant 2} a_{m-2, \alpha}(0,0) \partial^{\alpha} u_{m-2}(0)=f(0,0) .
$$

Since by the assumption $\sum_{|\alpha|=2}\left|a_{m-2},{ }_{\alpha}(0,0)\right| \neq 0, u_{m-2}(y)$ is not arbitrary, which proves the Theorem.

Next we consider the case where

$$
a_{m-1,0}(0,0) \neq 0 \text {. }
$$


On the initial-value problems with data on a double characteristic 363

For the sake of systematic treatment, by changing the notation, we write (1.4) in the following form:

$$
\sum_{i+|\nu| \leqslant m} a_{i \nu}(x, y) \partial_{x}^{i} \partial_{y}^{\nu} u+f(x, y)=0 .
$$

By expanding each coefficient $a_{i \nu}(x, y)$,

$$
a_{i \nu}(x, y)=\sum_{k \geqslant 0} a_{i \nu}^{(k)}(y) x^{k}
$$

and taking account of the assumption, we can assume without loss of generality that

$$
\begin{cases}1) & a_{m, 0}^{(0)}(y) \equiv a_{m, 0}^{(1)}(y) \equiv 0, \\ \text { 2) } & a_{m-1, \nu}^{(0)}(y) \equiv 0 \quad \text { for }|\nu|=1, \\ 3) & a_{m-1,0}^{(0)}(y) \equiv-1, a_{m-1,0}^{(k)}(y) \equiv 0 \quad \text { for } k \geqq 1 .\end{cases}
$$

Now we consider the formal solution of (3.6),

$$
u(x, y)=\sum_{p \geqslant m-2} u_{p}(y) x^{p} / p !
$$

We want to show that we can choose an initial data $u_{m-2}(y)$ in such a way that the formal solution (3.8) never converge in any neighborhood of the origin. At first, let us remark that the coefficient of $x^{q}$ of

$$
a_{i \nu}(x, y) \partial_{x}^{i} \partial_{y}^{\nu}\left(\sum_{p \geqslant m-2} u_{p}(y) x^{p} / p !\right)
$$

is

$$
\sum_{k \geqslant 0} a_{i \nu}^{(k)}(y) \partial^{\wedge} u_{q+i-k}(y) /(q-k) !
$$

where, in the summation, the terms corresponding to $q-k<0$, should be replaced by 0 .

Thus, taking account of (3.7), we have the following relations:

$$
\left\{\begin{array}{l}
u_{q+m-1} / q !=\sum_{k \geqslant 2} a_{m, 0}^{(k)}(y) u_{q+m-k} /(q-k) ! \\
\quad+\sum_{|\nu|=1} \sum_{k \geqslant 1} a_{m-1, \nu}^{(k)}(y) \partial^{\nu} u_{q+m-1-k} /(q-k) ! \\
\quad+\sum_{|\nu| \leqslant 2} \sum_{k \geqslant 0} a_{m-2, \nu}^{(k)}(y) \partial^{\nu} u_{q+m-2-k} /(q-k) !
\end{array}\right.
$$




$$
\mid \begin{aligned}
& +\sum_{\substack{i+|\nu| \leqslant m \\
i \leqslant m-3}} \sum_{k \geqslant 0} a_{i, \nu}^{(k)}(y) \partial^{\nu} u_{q+i-k} /(q-k) !+f_{q}(y) / q ! \\
& (q=0,1,2, \ldots) ; \text { where } f(x, y)=\sum_{p \geqslant 0} f_{p}(y) x^{p} / p ! .
\end{aligned}
$$

More precisely,

$$
\begin{aligned}
& u_{m-1}(y)=\sum_{|\nu| \leqslant 2} a_{m-2, \nu}^{(0)}(y) \partial^{\nu} u_{m-2}(y)+f_{0}(y) \\
& u_{m}(y)=\sum_{|\nu| \leqslant 2} a_{m-2, \nu}^{(0)}(y) \partial^{\nu} u_{m-1}(y)+\sum_{|\nu|=1} a_{m-1, \nu}^{(1)}(y) \partial^{\nu} u_{m-1}(y) \\
& +L_{3}^{(1)}(y, \partial) u_{m-2}(y)+f_{1}(y)
\end{aligned}
$$

$$
\left\{\begin{array}{l}
u_{q+m-1}(y)=\left(\sum_{|\nu| \leqslant 2} a_{m-2, \nu}^{(0)}(y) \partial^{\nu}+q \sum_{|\nu|=1} a_{m-1, \nu}^{(1)}(y) \partial^{\nu}\right. \\
\left.\quad+q(q-1) a_{m, 0}^{(2)}\right) u_{q+m-2} \\
+\sum_{j=3}^{q+2} L_{j}^{(q)}(y, \partial) u_{q+m-j}+f_{q},
\end{array}\right.
$$

$(q=0,1,2, \ldots)$, where $L_{j}^{(q)}(y, \partial)$ is differential operator of order $\leqslant j$. Now we can show, by induction on $q$, the following ralations:

$$
\begin{aligned}
& u_{q+m-1}(y)=\left(\sum_{|\nu|=2} a_{m-2, \nu}^{(0)}(0) \partial^{\nu}\right)^{q+1} u_{m-2}(y) \\
& +\mathscr{L}_{2 q+2}^{(q)}(y, \partial) u_{m-2}(y)+\widetilde{\mathscr{L}}_{2 q}^{(q)}(y, \partial)\left\{f_{0}, f_{1}, \ldots, f_{q}\right\}, \\
& (q=0,1,2, \ldots),
\end{aligned}
$$

where $\mathscr{L}_{2 q+2}^{(q)}$ is differential operator of order $2 q+2$, whose coefficients corresponding to the homogeneous part of order $2+2 q$ are all zero at the origin, and $\widetilde{\mathscr{L}}_{2 q}^{(q)}$ is also differential operator of order $\leqslant 2 q$.

By the assumption of the double characteristic, there exists an $\eta \in C^{n-1}$ such that

$$
\sum_{|\nu|=2} a_{m-2, \nu}^{(0)}(0) \eta^{\nu}=1
$$

Now we define

$$
u_{m-2}(y)=\sum_{p \geqslant 0} \alpha^{2 p} e^{i \theta_{2 p}}<y, \eta>^{2 p},
$$


On the initial-value problems with data on a double characteristic 365 where $\alpha(0<\alpha<1)$ is a fixed constant, and the arguments $\theta_{2 p}(p=0$, $1,2, \ldots)$ are defined recurrently in the following manner:

At first,

$$
\begin{aligned}
& \left(\sum_{|\nu|=2} a_{m-2, \nu}^{(0)}(0) \partial^{\nu}\right)^{q+1}\left(c x^{2 q+2} e^{i \theta_{2 q+2}}<y, \eta>^{2 q+2}\right) \\
& \quad=e^{i \theta_{2 q+2}} c \alpha^{2 q+2}(2 q+2) ! .
\end{aligned}
$$

Note that, since the coefficients of order $2 y+2$ of $\mathscr{L}_{2 q+2}^{(q)}(y, \partial)$ vanish at the origin, we see that $\mathscr{L}_{2 q+2}^{(q)}(0, \partial) u_{m-2}(0)$ depends only on the terms $\sum_{p=0}^{q} \ldots$ in (3.13). So we define $\theta_{2 q+2}$ by (assuming that $\theta_{0}, \theta_{2}, \ldots$, $\theta_{2 q}$ are already defined):

$$
\begin{aligned}
& \theta_{2 q+2}=\arg \left(\left.\mathscr{L}_{2 q+2}^{(q)}(0, \partial)\left(\sum_{p=0}^{q} \alpha^{2 p} e^{i \theta_{2 p}}<y, \eta>^{2 p}\right)\right|_{y=0}\right. \\
& \left.+\left.\widetilde{\mathscr{L}}_{2 q}^{(q)}\left\{f_{0}, f_{1}, \cdots f_{q}\right\}\right|_{y=0}\right) .
\end{aligned}
$$

Thus we have

$$
\begin{aligned}
& \left|u_{q+m-1}(0)\right| \geqslant\left|\left(\sum_{|\nu|=2} a_{m-2, \nu}^{(0)}(0) \partial^{\nu}\right)^{q+1}\left(\alpha^{2 q+2} e^{i \theta_{2 q+2}}<y, \eta>^{2 q+2}\right)\right|_{y=0} \mid \\
& =\alpha^{2 q+2}(2 q+2) ! .
\end{aligned}
$$

On the other hand,

$$
u(x, 0)=\sum_{p \geq m-2} u_{p}(0) x^{p} / p !
$$

Thus,

$$
\left|u_{p}(0) / p !\right| \geqslant \alpha^{2 p-2 m+4}(2 p-2 m+4) ! / p !
$$

This implies, by Stirling,

$$
p \sqrt{\left|u_{p}(0) / p !\right|} \sum \alpha^{2 p} \sqrt{(2 p) ! / p !} \rightarrow+\infty, \quad \text { when } p \rightarrow+\infty .
$$

Thus, (3.17) is not convergent in any neighborhood of the origin, which proves the Theorem.

\section{§4. Proof of Theorem 2}

At first we consider the following fairly simple equation: 


$$
\begin{aligned}
& x \partial_{x}^{m} u+\partial_{x}^{m-1} u=c x^{2} \partial_{x}^{m} u+x \sum_{\substack{i \leqslant m-1 \\
i+|\nu| \leqslant m}} a_{i \nu}(y) \partial_{x}^{i} \partial_{y}^{\nu} u \\
& \quad+\sum_{\substack{i \leqslant m-2 \\
i+|\nu| \leqslant m}} b_{i \nu}(y) \partial_{x}^{i} \partial_{y}^{\nu} u+f(y),
\end{aligned}
$$

where $c$ is a constant and $m \geqslant 2$; all the coefficients are assumed to be analytic, more precisely,

$$
\left|\partial^{\alpha} a_{i \nu}(y)\right|,\left|\partial^{\alpha} b_{i \nu}(y)\right| \leqslant \frac{|\alpha| !}{(3 \rho)^{|\bar{\alpha}|}} M \quad \text { for }|y| \leqslant a .
$$

Let us consider the following initial-value problem

$$
\partial_{x}^{i} u(0, y)=0, \quad i=0,1,2, \ldots, m-2 .
$$

We shall show

Lemma. The problem (4.1)-(4.3) has a unique solution $u(x, y)$ in a neighborhood of the origin.

Proof. Let

$$
u(x, y)=\sum_{p \geqslant m-1} u_{p}(y) x^{p} / p !
$$

be the formal solution of (4.1)-(4.3). Then we have

$$
\begin{gathered}
(q+1) u_{q+m-1}=q(q-1) c u_{q+m-2}+q_{\substack{i \leqslant m-1 \\
i+|\nu| \leqslant m}} a_{i \nu}(y) \partial^{\nu} u_{q+i-1} \\
+\sum_{\substack{i \leqslant m-2 \\
i+|\nu| \leqslant m}} b_{i \nu}(y) \partial^{\nu} u_{q+i}, \quad q=1,2, \ldots, \\
u_{m-1}(y)=f(y) .
\end{gathered}
$$

We assume that

$$
\left|\partial^{\alpha} f(y)\right| \leqslant \frac{|\alpha| !}{\rho^{|\alpha|}} A
$$

and we claim that the following estimates

$$
\left|\partial^{\alpha} u_{p}(y)\right| \leqslant \frac{(2 p+|\alpha|) !}{p !} C^{3 p+|\alpha|} A
$$


On the initial-value problems wilh data on a double characteristic 367 hold for any $\alpha$ and for any $p(\geqslant m-1)$, if we choose the constant $C$ sufficiently large. We show this by induction on $p$. At first we see that (4.6) is true for $p=m-1$ if we choose $C \geqslant \max (1,1 / \rho)$; we assume that (4.6) is true for $p=m-1, m, \ldots, q+m-2$, and we show that (4.6) is true for $p=q+m-1$. For this purpose, we use the following lemma due to Mizohata $([4]$, p. 275): Let $a(x)$ and $b(x)$ be analytic functions satisfying

$$
\begin{aligned}
& \left|\partial^{\nu} a(x)\right| \leqslant \frac{(r+|\nu|) !}{(k \rho)^{|\nu|}} A, \quad(k>1) \\
& \left|\partial^{\nu} b(x)\right| \leqslant \frac{(s+|\nu|) !}{\rho^{|\nu|}} B
\end{aligned}
$$

where $r$ and $s$ are non-negative integers, then

$$
\left|\partial^{\nu}\{a(x) b(x)\}\right| \leqslant \frac{(r+s+|\nu|) !}{\rho^{|\nu|}}(k / k-1) A B / C_{r}^{r+s} .
$$

Let us remark that, in the actual case, we shall use this lemma only for $r=0$ and $k=3$, so the last estimates become:

$$
\left|\partial^{\nu}\{a(x) b(x)\}\right| \leqslant \frac{(s+|\nu|) !}{\rho^{|\nu|}} \frac{3}{2} A B .
$$

This remarked, under the assumption $C \geqslant \max (1,1 / \rho)$, we have

$$
\begin{aligned}
& \left|\partial^{\alpha}\left\{a_{i \nu}(y) \partial_{y}^{\nu} u_{q+i-1}\right\}\right| \leqslant \frac{(2 q+2 i-2+|\nu|+|\alpha|) !}{(q+i-1) !} \\
& \quad \times C^{3 q+3 i-3+|\nu|+|\alpha|} \frac{3}{2} A M .
\end{aligned}
$$

Since $i+|\nu| \leqslant m$, the right hand side is estimated by

$$
\frac{(2 q+i-2+m+|\alpha|) !}{(q+i-1) !} C^{3 q+2 i-3+m+|\alpha|} \frac{3}{2} A M .
$$

On the other hand,

$$
\frac{(2 q+m-2+i+|\alpha|) !}{(q+i-1) !}=\frac{(2 q+2 m-2+|\alpha|) !}{(q+m-1) !}
$$




$$
\begin{aligned}
& \times \frac{(q+m-1) \ldots \ldots \ldots \ldots(q+i)}{(2 q+2 m-2+|\alpha|) \ldots \ldots(2 q+m-1+i+|\alpha|)} \\
\leqslant & \frac{1}{2^{m-i}} \frac{(2 q+2 m-2+|\alpha|) !}{(q+m-1) !}
\end{aligned}
$$

Since $i \leqslant m-1$, we obtain finally,

$$
\begin{aligned}
& \left|\partial^{\alpha}\left\{a_{i \nu}(y) \partial^{\nu} u_{q+i-1}\right\}\right| \\
& \quad \leqslant \frac{(2 q+2 m-2+|\alpha|) !}{(q+m-1) !} C^{3(q+m-1)+|\alpha|}\left(\begin{array}{l}
3 \\
2
\end{array} \frac{M}{C}\right) A .
\end{aligned}
$$

In the similar way, we get

$$
\begin{aligned}
& \left|\partial^{\alpha}\left\{b_{i \nu}(y) \partial^{\nu} u_{q+i}\right\}\right| \leqslant \frac{(2 q+2 i+|\nu|+|\alpha|) !}{(q+i) !} C^{3 q+3 i+|\nu|+|\alpha|} \times \frac{3}{2} M A \\
& \leqslant \frac{(2 q+m+i+|\alpha|) !}{(q+i) !} C^{3 q+2 i+m+|\alpha|} \times \frac{3}{2} A M .
\end{aligned}
$$

Since $i \leqslant m-2$, this last expression is majorized again by

$$
\frac{(2 q+2 m-2+|\alpha|) !}{(q+m-2) !} C^{3(q+m-1)+|\alpha|} C^{-1} \times \frac{3}{2} M A .
$$

Thus we get

$$
\begin{aligned}
& \frac{1}{q+1}\left|\partial^{\alpha}\left\{b_{i \nu}(y) \partial^{\nu} u_{q+i}\right\}\right| \leqslant \frac{(2 q+2 m-2+|\alpha|) !}{(q+m-1) !} C^{3(q+m-1)+|\alpha|} \\
& \quad \times\left(\frac{3}{2} m \frac{M}{C}\right) A
\end{aligned}
$$

Finally,

$$
\left\{\begin{array}{l}
\frac{q(q-1)}{q+1}\left|\partial^{\alpha} u_{q+m-2}\right| \leqslant q \frac{(2 q+2 m-4+|\alpha|) !}{(q+m-2) !} C^{3(q+m-2)+|\alpha|} A \\
\leqslant \frac{(2 q+2 m-2+|\alpha|) !}{(q+m-1) !} C^{3(q+m-1)+|\alpha|}(1 / 2 C) A
\end{array}\right.
$$

The above estimates (4.7), (4.8), (4.9) show that (4.6) is true for $p=q+m-1$, if we choose $C$ large, namely for example, if $C \geqslant n^{m}$ $\times \max (1,1 / \rho, 3 m M, c)$. 
On the initial-value problems with data on a double characteristic 369

From (4.6), we see easily that the formal solution $u(x, y)$ defined by (4.4) has the estimates of the form

$$
\left|\partial_{x}^{q} \partial_{y}^{\alpha} u(x, y)\right| \leqslant(q+|\alpha|) ! C^{\prime q+|\alpha|} A^{\prime},
$$

for $|x| \leqslant \delta,|y| \leqslant a$ if we choose $\delta$ small, and $C^{\prime}, A^{\prime}$ large.

The proof is thus complete.

\section{Proof of Theorem 2.}

Without loss of generality, we can assume that the initial data are all zero. For the proof we consider the following equation, equivalent to (1.4):

$$
\begin{aligned}
& x \partial_{x}^{m} u+a(y) \partial_{x}^{m-1} u=x \sum_{|\alpha| \leqslant \mid} a_{m-1, \alpha}(x, y) \partial_{x}^{m-1} \partial_{y}^{\alpha} u \\
& \quad+\sum_{\substack{i \leqslant m-2 \\
i+|\alpha| \leqslant m}} a_{i, \alpha}(x, y) \partial_{x}^{i} \partial_{y}^{\alpha} u+f(x, y) .
\end{aligned}
$$

Then the assumption in the Theorem means

$$
a(0) \notin\{0,-1,-2, \ldots\} .
$$

Let us consider the formal solution

$$
u(x, y)=\sum_{p \geqslant m-1} u_{p}(y) x^{p} / p !
$$

Since the uniqueness is obvious, we have only to show the convergence and the analyticity of (4.11).

In view of the recurrence formulas between $u_{m-1}, u_{m}, \ldots$, we choose a majorant $A(y)$ such that

$$
\frac{1}{n-1+a(y)}<\frac{1}{n} A(y),
$$

for all positive integers. Such a function exists, because if we choose $\delta$ small, then in the complex domain $\left|\eta_{i}\right| \leqslant \delta \quad(i=1, \ldots, n-1)$, $\sup _{\eta}\left|\frac{n}{n-1+a(\eta)}\right|$ remains bounded (with respect to $n$ ).

Now let $M /\left(1-\frac{x}{\rho}\right)\left(1-\frac{y_{1}+\cdots+y_{n-1}}{\rho}\right)$ be a common majorant of 
$a_{m-1, \alpha}(x, y), a_{i, \alpha}(x, y)$ and $f(x, y)$, then the formal solution

$$
U(x, y)=\sum_{p \geq m-1} U_{p}(y) x^{p} / p !
$$

of the equation

$$
\begin{aligned}
& x \partial_{x}^{m} U+\partial_{x}^{m-1} U=A(y) \frac{M}{\left(1-\frac{x}{\rho}\right)\left(1-\frac{y_{1}+y_{2}+\cdots+y_{n-1}}{\rho}\right)} \\
& \quad \times\left(x \sum_{|\alpha| \leqslant 1} \partial_{x}^{m-1} \partial_{y}^{\alpha} U+\sum_{\substack{i \leqslant m-2 \\
i+|\alpha| \leqslant m}} \partial_{x}^{i} \partial_{y}^{\alpha} U+1\right)
\end{aligned}
$$

is a majorant of (4.11). More precisely,

$$
u_{p}(y) \ll U_{p}(y) \quad(p=m-1, m, \ldots) .
$$

On the other hand, (4.12) is a special case of (4.1). In fact, this equation can be written in the form

$$
\left(1-\frac{x}{\rho}\right)\left(x \partial_{x}^{m}+\partial_{x}^{m-1}\right) U=B(y)\left(x \sum_{|\alpha| \leqslant 1} \partial_{x}^{m-1} \partial_{y}^{\alpha} U+\sum_{\substack{i \leqslant m-2 \\ i+|\alpha| \leqslant m}} \partial_{x}^{i} \partial_{y}^{\alpha} U+1\right) .
$$

Owing to the Lemma, we see that $U(x, y)$ represents a convergent series in a neighborhood of the origin, this proves that $u(x, y)$ is an analytic solution of (4.10) in the neighborhood of the origin.

\section{§5. Proof of Theorem 3}

We consider the following formal solution of (1.4),

$$
u(x, y)=\sum_{p=0}^{\infty} u_{p}(y) x^{p} / p !
$$

Using (1.4), (5.1) we see easily that (2.1) is a necessary condition.

Taking account of the assumption of theorem we see that $u_{m-1}$, $u_{m}, \ldots, u_{m+p_{0}-2}$ are determined by the initial data (1.6). If $u_{m+p_{0}-1}$ is given, $u_{m+p_{0}}, u_{m+p_{0}+1}, \ldots$ are determined. We will show that the convergence of (5.1), if initial data (1.6) satisfy (2.1) and $u_{m+p_{0}-1}$ is given. 
On the initial-value problems with data on a double characteristic 371

For the proof we use the following equation (5.2) instead of (1.4).

$$
\begin{aligned}
& x \partial_{x}^{m} u-p_{0} \partial_{x}^{m-1} u=x \sum_{|\alpha| \leqslant 1} a_{m-1, \alpha}(x, y) \partial_{x}^{m-1} \partial_{y}^{\alpha} u \\
& \quad+\sum_{\substack{i \leqslant m-2 \\
i+|\alpha| \leqslant m}} a_{i, \alpha}(x, y) \partial_{x}^{i} \partial_{y}^{\alpha} u+f(x, y) .
\end{aligned}
$$

The initial data are

$$
\partial_{x}^{i} u(0, y)=u_{i}(y) \quad i=0,1, \ldots, m-2 .
$$

The formal solution is

$$
u(x, y)=\sum_{p=0}^{\infty} u_{p}(y) x^{p} / p !
$$

Using (5.2), (5.4) we obtain

$$
\begin{gathered}
\frac{\left(q-m-p_{0}+1\right)}{(q-m+1) !} u_{q}(y)=\sum_{|\alpha| \leqslant 1} \sum_{p+j=q-1} a_{m-1, \alpha}^{(j)}(y) \frac{1}{(p-m+1) !} \partial_{y}^{\alpha} u_{p}(y) \\
+\sum_{\substack{i \leqslant m-2 \\
i+|\alpha| \leqslant m}} \sum_{p+j=q-m+1+i} a_{i, \alpha}^{(j)}(y) \frac{1}{(p-i) !} \partial_{y}^{\alpha} u_{p}(y)+f^{(q-m+1)}(y) \\
q \geqslant m-1
\end{gathered}
$$

where $a_{j, \beta}(x, y)=\sum_{k=0}^{\infty} a_{j, \beta}^{(k)}(y) x^{k}, f(x, y)=\sum_{k=0}^{\infty} f^{(k)}(y) x^{k}$.

We account of the following initial value problem (5.6)-(5.7).

$$
\begin{gathered}
x \partial_{x}^{m+p_{0}+1} u+\left(p_{0}+1\right) \partial_{x}^{m+p_{0}} u-p_{0} \partial_{x}^{m+p_{0}} u \\
=\partial_{x}^{p_{0}+1}\left[x \sum_{|\alpha| \leqslant 1} a_{m-1, \alpha}(x, y) \partial_{x}^{m-1} \partial_{y}^{\alpha} u\right. \\
\left.\quad+\sum_{\substack{i \leqslant m-2 \\
i+|\alpha| \leqslant m}} a_{i, \alpha}(x, y) \partial_{x}^{i} \partial_{y}^{\alpha} u+f(x, y)\right] \\
\partial_{x}^{i} u(0, y)=u_{i}(y) \quad i=0,1, \ldots, m+p_{0}-1 \\
\text { when } i=0,1, \ldots, m-2, u_{i}(y) \text { is the same as (5.3), } \\
\text { when } i=m-1, \ldots, m+p_{0}-2, u_{i}(y) \text { is that we obtain } \\
\text { by (5.5), and } u_{m+p_{0}-1}(y) \text { is arbitraly. }
\end{gathered}
$$

The formal solution of the initial value problem (5.6)-(5.7) converges by theorem 2. and this formal solution coincides with the formal 
solution corresponding to the initial value problem (5.2)-(5.3). q.e.d.

At the end, the author wishes to thank Prof. Mizohata, for his valuable suggestions.

\section{Kyoto UNIVERSITY}

\section{Reference}

[1] E. Goursat: Cours d'analyse mathématique, tome II (1949)

[2] J. Hadamard: Lectures on Cauchy's problem in linear partial differential equations, (1952)

[3] L. Hörmander: Linear partial differential operators, (1964)

[4] S. Mizohata: Solutions nulles et solutions non analytiques. J. Math. KyotoUniv. 1 (1962), p. 271-302.

[5] J. Vaillant: Données de Cauchy portées par une caractéristique double, ....., Séminaire sur les équations aux dérivées partielles, Collège de France, 196667, p. 27-63. 